\title{
Alteraciones del neurodesarrollo en pacientes con hipotiroidismo congénito: Recomendaciones para el seguimiento
}

\author{
ALICIA NÚÑEZ ${ }^{1}$, PAULA BEDREGAL ${ }^{2}$, \\ CARLOS BECERRA ${ }^{3}$, FRANCISCA GROB L. ${ }^{4}$
}

\section{Neurodevelopmental assessment of patients with congenital hypothyroidism}

\begin{abstract}
Congenital hypothyroidism $(\mathrm{CH})$ is the most common cause of preventable cognitive disability worldwide. Generally, it is produced by an alteration in the embryogenesis of the thyroid gland or by an alteration in the synthesis of thyroid hormones, which determine that affected patients have low or absent thyroid hormone concentrations. The importance of this fact is that brain development during the first three years of life is highly dependent on thyroid hormones. Prior to the implementation of national neonatal screening programs around the world, 8 to $27 \%$ of children with $\mathrm{CH}$ had an IQ lower than 70 . Nowadays, this percentage is close to 0 in countries that have implemented the program. In Chile, CH neonatal screening program achieved national coverage in 1996. Currently, the incidence of the disease in our country is 1: 3163. The degree of disability produced by $\mathrm{CH}$ not only depends on the time of detection of the disease and the prompt start of therapy, but also on an adequate monitoring. Despite screening programs, neurocognitive impairment in schoolchildren and teenagers with $\mathrm{CH}$ is still observed, reflected in lower scores in cognitive, language and gross motor assessments, receptive communication, expressive communication, fine motor and gross motor skills compared to healthy children. Also, lesser achievements in learning and language disorders are observed. The objective of this review is to update the information available on neurodevelopment of patients with $\mathrm{CH}$.

(Rev Med Chile 2017; 145: 1579-1587)

Key words: Cognitive Dysfunction; Congenital Hypothyroidism; Neurodevelopmental Disorders.
\end{abstract}

E 1 hipotiroidismo congénito (HC) es la principal causa de discapacidad cognitiva prevenible en el mundo. Generalmente, se produce por una alteración en la embriogénesis de la glándula tiroides o por una alteración en la síntesis de las hormonas tiroideas, lo que determina que los pacientes afectados presentan concentraciones bajas o ausentes de hormonas tiroideas. La importancia de este hecho radica en que el desarrollo cerebral durante los primeros 3 años de vida es altamente dependiente de hormonas tiroideas.
'División de Pediatría, Unidad de Neurología Pediátrica, Pontificia Universidad Católica. Santiago, Chile.

${ }^{2}$ Departamento de Salud Pública, Pontificia Universidad Católica.

Santiago, Chile.

${ }^{3}$ Departamento Ciclo Vital. División de Prevención y Control de Enfermedades. Ministerio de Salud. Santiago, Chile. ${ }^{4}$ División de Pediatría, Unidad de Endocrinología Pediátrica, Pontificia Universidad Católica. Santiago, Chile.

Declaración de conflicto de interés: Los autores declaran no tener conflicto de intereses Financiamiento: Este trabajo fue apoyado por FONIS (Fondo Nacional de Investigación y Desarrollo en Salud) (SA14ID0038), una beca de investigación de la Comisión Nacional de Investigación Científica y Tecnológica del Ministerio de Educación del Gobierno de Chile (a F.G).

Recibido el 3 de junio de 2016, aceptado el 2 de marzo de 2017.

Correspondencia a:

Francisca Grob Lunecke Pontificia Universidad Católica de Chile. Escuela de Medicina, División de Pediatría. Lira 85, $5^{\circ}$ piso, Santiago. Código Postal: 8330074. fgrob@med.puc.cl
La pesquisa neonatal de $\mathrm{HC}$ es en la actualidad uno de los programas de salud pública más difundidos en el mundo. Las consecuencias devastadoras potenciales e irreversibles del HC para el desarrollo intelectual han sido reconocidas por décadas y ocurre en forma muy precoz luego del nacimiento, antes que los síntomas y signos clásicos del HC puedan ser reconocidos. Por esta razón, programas de tamizaje neonatal fueron creados en el mundo en la década de los 70, y al día de hoy son considerados como un estándar de salud en todos los países desarrollados. El objetivo 
de estos programas es el diagnóstico precoz de esta patología, que permita el inicio de tratamiento temprano con hormonas tiroideas sintéticas, o levotiroxina, para así evitar el compromiso neurológico. En 2010, la Asamblea Mundial de Salud reportó y adoptó una resolución que describe y promueve los componentes básicos para crear un programa nacional de vigilancia, prevención y cuidado de anomalías congénitas (que incluye aquellas metabólicas e $\mathrm{HC})^{5}$. La evaluación del resultado de estos programas a largo plazo ha demostrado que el desarrollo intelectual de los niños identificados durante el período de recién nacido es sustancialmente mejor que el de aquellos identificados en la época previa a los programas de tamizaje $^{6}$. A pesar de esto, existe $10 \%$ de los niños diagnosticados por los programas que aun así requerirá educación especial ${ }^{7}$. Este hecho probablemente refleja la heterogeneidad de los pacientes al momento del diagnóstico y factores relacionados al inicio del tratamiento y al seguimiento de la patología. En Chile, el programa de tamizaje neonatal de HC otorga cobertura a 100\% de los nacidos vivos en todo el territorio nacional y tiene como objetivo prevenir la discapacidad cognitiva que produce esta enfermedad si no es detectada y tratada desde el período neonatal. Después de 20 años de implementación, este programa se considera totalmente consolidado en todo el país en términos de organización, cobertura y control de calidad de los laboratorios ${ }^{8}$. En el período 1992-2012 se han realizado más de dos y medio millones de determinaciones y se ha confirmado el diagnóstico de más de 1.300 niños portadores de esta patología ${ }^{2}$.

El objetivo de este artículo es analizar la evidencia disponible en la literatura acerca del desarrollo neurológico y cognitivo en pacientes afectados por hipotiroidismo congénito y sobre la base de este análisis proponer recomendaciones de su evaluación y seguimiento.

\section{Rol de las hormonas tiroideas en el desarrollo del sistema nervioso central}

Las hormonas tiroideas influyen de manera crucial en el desarrollo del sistema nervioso desde los últimos meses de vida fetal y durante los dos primeros años de vida, participando en los procesos de mielinización, arborización dendrítica, de- sarrollo de sinapsis y migración neuronal ${ }^{9}$ (Figura 1). La síntesis de las hormonas tiroideas-tiroxina (T4) y triyodotironina (T3) -que resulta de la deyodinación de la T4- requiere de varios pasos, desde el transporte activo del yodo hacia el interior del tirocito, hasta su secreción fuera de éste. La T3 producida actúa en los diferentes tejidos objetivos a través del receptor nuclear de hormonas tiroideas, el que se encuentra unido a secuencias especiales de nucleótidos en las regiones promotoras de genes que son regulados de manera positiva o negativa por las hormonas tiroideas.

Para comprender por qué es importante mantener una adecuada concentración de hormonas tiroideas, debemos conocer su acción en el desarrollo cerebral. El tiroides fetal es capaz de secretar hormonas tiroideas alrededor de la semana 16 , por lo que durante el primer trimestre, la concentración de hormonas tiroideas fetales depende de la concentración de hormonas tiroideas maternas. La T4 fetal es transportada al cerebro, atraviesa la barrera hematoencefálica e ingresa a los astrocitos a través del transportador Oatp1c1 (T4-selective organic anion transporting polypeptide) localizado en su superficie. Aquí, la T4 es metabolizada intracelularmente por la deyodinasa tipo 2 a T3, $y$ así es transportada fuera del astrocito a través de otro transportador de membrana aún no identificado. Una vez en el intersticio del parénquima cerebral, la T3 es rápidamente internalizada por las neuronas y oligodendrocitos a través del transportador MCT8. Una vez dentro de estas células, la T3 puede unirse directamente al receptor de hormonas tiroideas, o ser metabolizada por la deyodinasa tipo 2 a T2 biológicamente inactiva. La unión de la T3 a su receptor lleva a un aumento o disminución en la transcripción de genes. La T4 también ejerce acciones no genómicas, relacionadas especialmente con la polimerización de neuronas, importante para su migración y formación de proyecciones ${ }^{10}$. En la corteza cerebral, las hormonas tiroideas regulan la expresión de la proteína Reelina, producida por las células de Cajal-Retzius de la capa 1. Esta proteína es esencial para la distribución de las neuronas corticales en capas. En el cerebelo, la migración de las células granulares de la capa germinal externa a la capa granular interna también necesita de las hormonas tiroideas en las últimas etapas. Las hormonas tiroideas participan además en el desarrollo de la sinaptogénesis, en parte mediante la formación 
Hipotiroidismo congénito y neurodesarrollo - A. Núñez et al

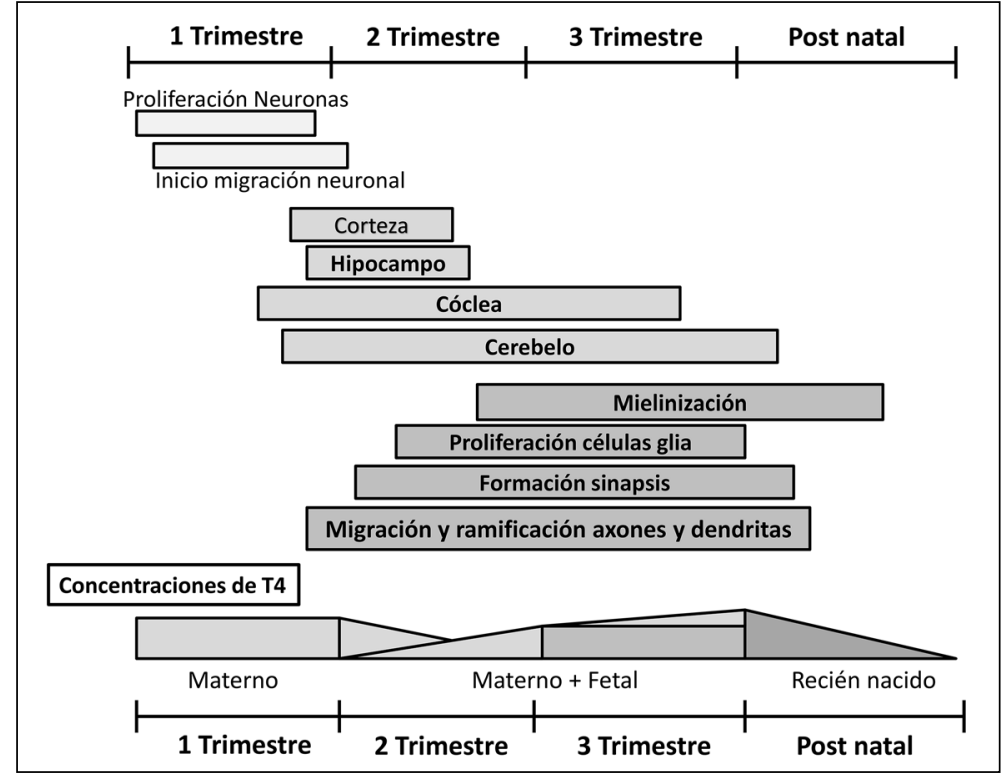

Figura 1. Desarrollo neurológico dependiente de hormonas tiroideas.

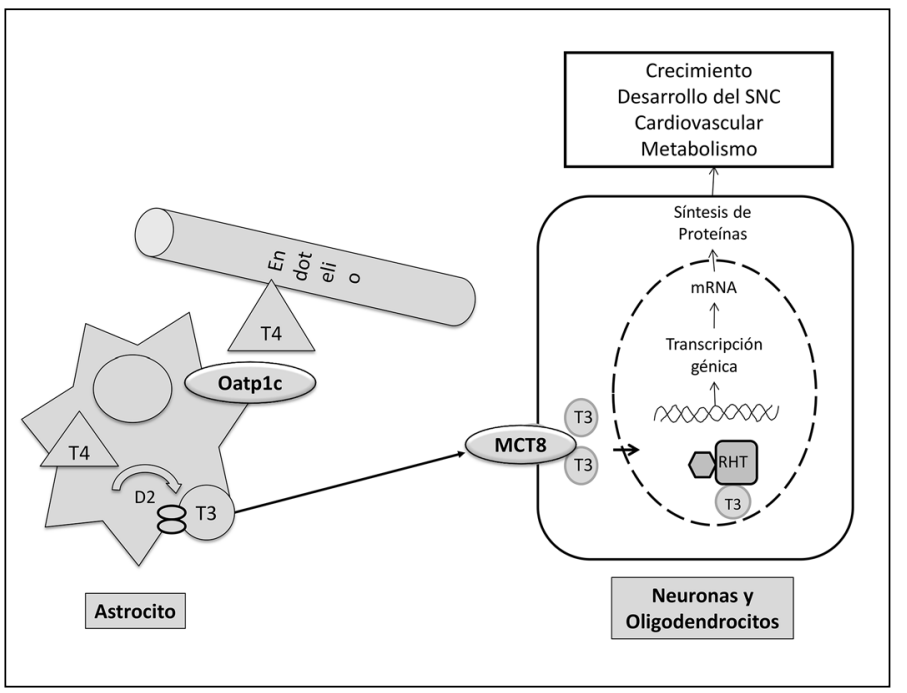

Figura 2. Hormonas tiroideas en el desarrollo neurológico del feto y recién nacido. La T4 es transportada al cerebro y atraviesa la BHE, siendo internalizada rápidamente a través del transportador Oatp1c1 al astrocito. En ese momento, la T4 es metabolizada intracelularmente por la Deyodinasa tipo 2 (D2) a T3, desde donde puede ser transportada fuera del astrocito por un transportador de membrana aun no identificado. Una vez en el intersticio del parénquima cerebral, la T3 es internalizada por las neuronas y oligodendrocitos a través del transportador MCT8. Una vez en la célula, la T3 puede unirse directamente al receptor de hormonas tiroideas, o ser metabolizada por la D2 a T2 biológicamente inactiva. La unión de la T3 a su receptor lleva a un aumento o disminución en la transcripción de genes. de arborizaciones dendríticas, por ejemplo, de las células de Purkinje del cerebelo, y de las células piramidales de la corteza, y también mediante el control de la expresión de proteínas de sinapsis, como la sinaptofisina. La mielinización es uno de los procesos clásicos regulados por las hormonas tiroideas, mediante acciones directas sobre la diferenciación de los oligodendrocitos y como consecuencia, la expresión de los genes de mielina ${ }^{11}$.

\section{Alteraciones del desarrollo neurológico y cognitivo en pacientes con HC}

Aunque la mayoría de los programa en países desarrollados logra los objetivos de iniciar el tratamiento a una edad y en una dosis adecuada, aún existen déficits neurológicos sutiles en pacientes evaluados durante el seguimiento, ya sea en la etapa preescolar o escolar los que presentan un 
menor coeficiente intelectual al compararlos con sus hermanos ${ }^{12}$. En estudios de seguimiento se ha visto que niños con $\mathrm{HC}$ severo tienen alteraciones intelectuales significativas al compararlas con la población normal, encontrándose puntajes cognitivos más bajos, mayor inatención y menor desempeño verbal al compararlos con población normal y con hipotiroidismo moderado ${ }^{13}$. Otros estudios descriptivos han observado menores puntuaciones en evaluaciones cognitivas, de lenguaje tanto receptivo como expresivo al comparar con población sana, además de puntajes más bajos en motricidad fina y motricidad gruesa ${ }^{3}$. También se han observado peores logros en aprendizaje y otras alteraciones del lenguaje ${ }^{4}$. Además, se ha sugerido que estos pacientes presentan compromiso de la memoria autobiográfica ${ }^{14}$ que estaría relacionado con la presencia de volúmenes hipocampales más pequeños ${ }^{15}$.

En el seguimiento hasta etapa adulta se ha visto que los mejores resultados en neurodesarrollo ocurre cuando el tratamiento con hormona tiroidea comienza antes de las 2 semanas de vida. Se han observado diferencias mínimas en coeficiente intelectual, desempeño escolar y test neuropsicológicos al compararlos con población sin HC. Las alteraciones más frecuentes incluyen alteración del procesamiento visoespacial, en la memoria selectiva y alteraciones sensoriomotrices. Al contrario, en pacientes que son tratados más tardíamente hay mayor riesgo de tener $\mathrm{CI}$ normal-lento, o algunos signos de alteración en pruebas neuropsicológicas las que incluyen alteración en habilidades aritméticas, lenguaje y coordinación motora ${ }^{16}$. Independiente de los factores de la enfermedad, coeficiente intelectual y habilidades motoras, son frecuentes las consecuencias negativas en términos de calidad de vida y autoestima en los niños y adultos con $\mathrm{HC}^{17}$. En esta etapa, se observa significativamente una mayor frecuencia de comorbilidades en el ámbito neuropsiquiátrico como compromiso de funciones cerebrales superiores, epilepsia o trastornos psiquiátricos, asociados a un moderado a severo retraso del desarrollo psicomotor ${ }^{18}$ así como a menores tasas de fecundidad en mujeres ${ }^{19}$.

Las alteraciones neurocognitivas observadas en los niños con HC identificados por el tamizaje neonatal dependen de factores modificables y no modificables, como factores relacionados con la severidad de la enfermedad y con el inicio de la terapia, otras variables tales como las influencias sociales, genéticas, ambientales y la calidad del manejo posterior. El nivel de educación parental, especialmente materno ${ }^{20}$, el nivel socioeconómi$\mathrm{CO}^{21}$ y el número de controles médicos durante el primer año de vida ${ }^{20}$, se han asociado de manera proporcional al resultado cognitivo de los pacientes afectados.

Dentro de los factores no modificables encontramos variables relacionadas con la gravedad del $\mathrm{HC}$ al momento del diagnóstico, definida como el grado de retraso de la edad ósea, los niveles de T4 o T4 libre y la etiología del HC. También factores socioculturales ${ }^{22}$, como la escolaridad de los padres $^{23} \mathrm{y}$ la ruralidad de las familias ${ }^{20}$ determinan la presencia de alteraciones neurocognitivas en el seguimiento. Se ha demostrado que los niños afectados con las formas más severas de la enfermedad, presentan una peor evolución neurológi$\mathrm{Ca}^{24,25}$, a pesar de haber iniciado el tratamiento en tiempos correctos y con dosis adecuadas ${ }^{26}$, lo que probablemente refleje el grado de hipotiroidismo intrauterino.

Dentro de los factores modificables que influencian el desarrollo cognitivo, se ha observado que la pesquisa y el inicio precoz del tratamiento, como la edad y dosis en que se inicia la terapia modifican el neurodesarrollo ${ }^{6,27,28}$. Se ha demostrado que se pueden lograr mejores resultados con el tratamiento inicial con una dosis alta de levotiroxina (10 a 15 ug/kg/día) para normalizar más rápidamente la función tiroidea ${ }^{29-31}$, dosis actualmente recomendada por el programa nacional de tamizaje de $\mathrm{HC}^{8}$. Sin embargo, el tratamiento con dosis altas de levotiroxina se asocia con episodios en que los niveles de T4 pueden aumentar en forma suprafisiológica, lo que puede provocar déficit en el estado de alerta y en el control inhibitorio ${ }^{32}$, alteraciones del temperamento ${ }^{33}$ y problemas de atención, comportamiento y psiquiátricos durante la infancia ${ }^{34}$. Es por esta razón que un seguimiento exhaustivo y frecuente se hace imperativo, y ya ha sido demostrado que un mayor número de evaluaciones durante los primeros años de vida se correlaciona con mejores resultados de neurodesarrollo ${ }^{20,31}$.

La evidencia demuestra que todos los pacientes diagnosticados por el programa con $\mathrm{HC}$ deben tener asegurado no solo el inicio de la terapia, sino que también una monitorización adecuada y permanente, especialmente durante los primeros 3 años de vida, para evitar períodos de sub o 
sobre-tratamiento. De esta manera, se evitaría el riesgo de presentar algunas de las alteraciones previamente mencionadas en su etapa escolar o adulta. Por lo anterior la evaluación del desarrollo psicomotor en forma sistemática en pacientes con HC es mandatoria para determinar en forma precoz alteraciones sutiles. Para esto, existe una gran diversidad de test neuropsicológicos que permiten evaluar el neurodesarrollo de estos pacientes.

\section{Los instrumentos de valoración del desarrollo e hipotiroidismo congénito}

La valoración del desarrollo en los servicios clínicos del sector público de salud de Chile, obedecen a las normas desarrolladas por el Ministerio de Salud. En 1979, la evaluación fue incorporada al Programa de Salud del Niño, y exclusivamente en aquellos menores de 2 años. El método de pesquisa utilizado fue el instrumento Escala de Evaluación del Desarrollo Psicomotor de 0-24 meses (EEDP), elaborado en Chile, y con normas nacionales ${ }^{35}$.

A partir de 1993, el enfoque estuvo centrado en la pesquisa temprana de trastornos severos. En 2004, se publicaron las Normas Técnicas de Estimulación y Evaluación del Desarrollo Psicomotor del niño y la niña menor de 6 años (2004), que recomienda: 1) Educación y capacitación a las familias y la comunidad en temas relacionados con desarrollo; 2) Aplicación de escalas de evaluación a los 2, 8, 18, 36 meses y a los 4 años; 3) Aplicación de una pauta breve que estandariza la observación del desarrollo del niño en la consulta, cuya construcción se basó en ítems de las pruebas estandarizadas en uso. En dichas normas la aplicación de EEDP se mantiene y se adiciona el Test de Desarrollo Psicomotor 2-5 años (TEPSI) ${ }^{36}$. Actualmente, la Norma Técnica para la supervisión de niños y niñas de 0 a 9 años en atención primaria y el Manual para el apoyo y seguimiento del desarrollo psicosocial de los niños y niñas de 0 a 6 años, continua promoviendo las mismas pruebas. La norma chilena del programa de $\mathrm{HC}^{8}$ recomienda realizar evaluación neurológica a los $3,12,18$ meses de vida y luego en forma anual; $y$ realizar evaluación psicológica con test de Bayley a los 4, 12, 18 meses de edad, luego una vez al año hasta los 42 meses de edad. En niños mayores de 42 meses, se recomienda utilizar la prueba de inteligencia Stanford-Binet en forma anual o cada 2 años hasta la adolescencia. La prueba de Stanford-Binet se creó con el objetivo de generar un instrumento para evaluar futuro desempeño en el proceso de escolarización de niños y niñas. La actual quinta versión arroja un CI. Esta prueba se utilizó en Chile (en su tercera versión) en el seguimiento de algunos pacientes con hipotiroidismo o fenilcetonuria, sin embargo se descontinuó su uso debido a la falta de estandarización para Chile de las nuevas versiones. Actualmente se recomienda usar Stanford-Binet o WPPSSI/WISC ${ }^{37}$.

En el mundo, existen múltiples escalas en uso. En Latinoamérica, al igual que en Chile, se está revisando cuál es el instrumento más pertinente de utilizar en los servicios de salud y educación inicial. Dentro de las escalas en evaluación de uso se encuentra PRUNAPE ${ }^{38}$, Inventario de Desarrollo Battelle, Escala abreviada del desarrollo de Nelson Ortíz ${ }^{39}$, Test de aprendizaje y desarrollo infantil TADI ${ }^{40}$, entre otras.

La situación en investigación muestra que los instrumentos más usados para valorar el desarrollo en niños y niñas se presentan en la Tabla 1. Adicionalmente se ha utilizado el desempeño escolar en matemáticas y lenguaje como una aproximación a las habilidades cognitivas. En Chile, adicionalmente se ha utilizado el inventario de desarrollo Battelle (en su versión española, adaptada en lenguaje a Chile). De las pruebas de la tabla, DENVER-II ha sido discontinuada su uso en Estados Unidos, pues no cumpliría con los criterios que actualmente se solicitan a las pruebas de desarrollo. Si bien se encuentra disponible a petición directa con los autores, no se recomienda su uso ${ }^{41}$. En el área de lenguaje se han utilizado las subescalas disponibles en aquellas pruebas de desarrollo global.

\section{Recomendaciones}

Dado que los pacientes con HC pueden presentar alteraciones neurocognitivas principalmente en las áreas motora, de inteligencia, memoria y de lenguaje, se sugiere evaluar el neurodesarrollo en forma periódica con distintos instrumentos que abarquen esas áreas (Tabla 2).

La primera evaluación por neurólogo debe realizarse al momento del diagnóstico, luego con frecuencia trimestral durante el primer año, cada 6 meses durante el segundo y tercer año y anual hasta los 6 años de tal forma de detectar alteraciones 


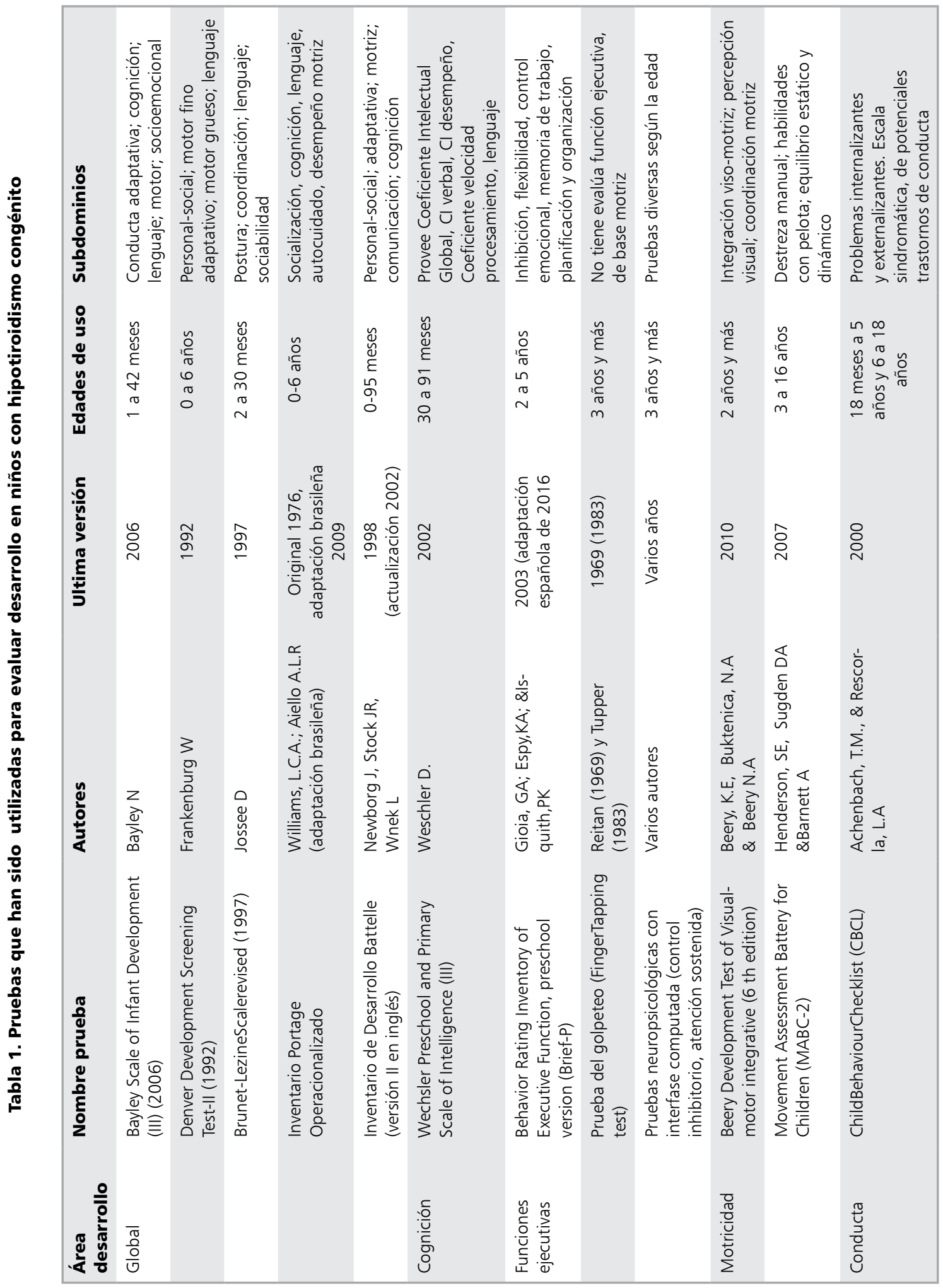


Tabla 2. Secuencia sugerida de evaluación de desarrollo en niños con HC

\begin{tabular}{|c|c|c|c|c|c|c|c|c|c|c|}
\hline & $\begin{array}{c}\text { Recién } \\
\text { nacido a } \\
3 \text { meses }\end{array}$ & $\begin{array}{c}2 \\
\text { meses }\end{array}$ & $\begin{array}{c}6 \\
\text { meses }\end{array}$ & $\begin{array}{c}9 \\
\text { meses }\end{array}$ & $\begin{array}{c}12 \\
\text { meses }\end{array}$ & $\begin{array}{c}18 \\
\text { meses }\end{array}$ & $\begin{array}{c}24 \\
\text { meses }\end{array}$ & $\begin{array}{c}36 \\
\text { meses }\end{array}$ & $\begin{array}{l}4 \text { y } 5 \\
\text { años }\end{array}$ & $\begin{array}{l}6 \text { a } 10 \\
\text { años }\end{array}$ \\
\hline Evaluación neurológica & $x$ & & $x$ & $x$ & $x$ & $x$ & $x$ & $x$ & $x$ & $x$ \\
\hline Test de Bayley o Battelle & & $x$ & $x$ & & $x$ & $x$ & $x$ & $x$ & & \\
\hline $\begin{array}{l}\text { WPPSI - Wechsler Preschool and } \\
\text { Primary Scale of Intelligence }\end{array}$ & & & & & & & & & $x$ & \\
\hline WISC & & & & & & & & & & $x$ \\
\hline
\end{tabular}

del neurodesarrollo en las distintas áreas. Además debe complementarse la evaluación neurológica con la medición de desarrollo psicomotor a través de test de Bayley a partir de los 2 meses de vida luego a los 6 meses y posteriormente semestral para detectar tempranamente alteraciones sutiles en las distintas áreas. El objetivo de la evaluación precoz y periódica con test de Bayley durante los primeros 2 años de vida es detectar anomalías en las distintas áreas del desarrollo psicomotor que pueden no ser evidenciadas al examen clínico neurológico ${ }^{3}$. Después de los 36 meses se sugiere evaluar con WPSSI y sobre los 6 años con WISC en forma anual.

Si en cualquiera de las evaluaciones antes de los 36 meses se observa algún grado de compromiso, la sugerencia es derivar a evaluación inmediata por neurólogo, además de la derivación oportuna a programa de atención temprana el que debería contar con fonoaudiólogo, terapeuta ocupacional y/o kinesiólogo dependiendo del área alterada. En estos casos, la frecuencia del seguimiento neurológico debe aumentar dependiendo de los hallazgos, además de complementar el estudio con neuroimágenes, evaluación neuropsicológica y otros estudios (sensoriales: visión, audición) como es pertinente en todo niño que presenta riesgo de retraso en neurodesarrollo. El objetivo de realizar derivación a atención temprana en niños con $\mathrm{HC}$ en los que se detecte alguna alteración es fundamentalmente optimizar los procesos de desarrollo cerebral en el período de mayor neuroplasticidad ya que esto influiría en los resultados en el neurodesarrollo a largo plazo ${ }^{42}$.

Agradecimientos: Agradecemos a todos los médicos que han contribuido al diagnostico y seguimiento de pacientes con hipotiroidismo congénito en Chile.

\section{Referencias}

1. Grosse SD, Van Vliet G. Prevention of intellectual disability through screening for congenital hypothyroidism: how much and at what level? Arch Dis Child 2011; 96 (4): 374-9.

2. Cornejo V, Raimann E, Cabello JF, Valiente A, Becerra C, Opazo M, et al. Past, present and future of newborn screening in Chile. J Inherit Metab Dis 2010; 33 Suppl 3: S301-6.

3. Komur M, Ozen S, Okuyaz C, Makharoblidze K, Erdogan S. Neurodevelopment evaluation in children with congenital hypothyroidism by Bayley-III. Brain \& development 2013; 35 (5): 392-7.

4. Bargagna S, Canepa G, Costagli C, Dinetti D, Marcheschi M, Millepiedi S, et al. Neuropsychological follow-up in early-treated congenital hypothyroidism: a problem-oriented approach. Thyroid : official journal of the American Thyroid Association 2000; 10 (3): 243-9.

5. Tarim OF, Yordam N. Congenital hypothyroidism in Turkey: a retrospective evaluation of 1000 cases. Turk J Pediatr 1992; 34 (4): 197-202.

6. Heyerdahl S, Oerbeck B. Congenital hypothyroidism: developmental outcome in relation to levothyroxine treatment variables. Thyroid: official journal of the American Thyroid Association 2003; 13 (11): 1029-38.

7. Tillotson SL, Fuggle PW, Smith I, Ades AE, Grant DB. Relation between biochemical severity and intelligence in early treated congenital hypothyroidism: a threshold effect. BMJ 1994; 309 (6952): 440-5.

8. Salud Md. Normas para el óptimo desarrollo de programas de búsqueda masiva de fenilquetonuria $\mathrm{e}$ hipotiroidismo congénito y otros errores innatos del 
metabolismo. 2007. Available from: http://web.minsal. cl/portal/url/item/dd7c4cf4c184c58de040010165016b2a. pdf.

9. Manriquez OM, Nagel BL, Vivanco WX. Evaluación neurológica en pacientes con hipotiroidismo congénito diagnosticado por rastreo neonatal. Revista chilena de pediatría 1998; 69: 56-9.

10. Patel J, Landers K, Li H, Mortimer RH, Richard K. Thyroid hormones and fetal neurological development. The Journal of endocrinology 2011; 209 (1): 1-8.

11. Bernal J, Guadano-Ferraz A, Morte B. Perspectives in the study of thyroid hormone action on brain development and function. Thyroid : official journal of the American Thyroid Association 2003; 13 (11): 1005-12.

12. Rovet JF. Children with congenital hypothyroidism and their siblings: do they really differ? Pediatrics 2005; 115 (1): e52-7.

13. Selva KA, Harper A, Downs A, Blasco PA, Lafranchi $\mathrm{SH}$. Neurodevelopmental outcomes in congenital hypothyroidism: comparison of initial T4 dose and time to reach target T4 and TSH. J Pediatr 2005; 147 (6): 77580.

14. Willoughby KA, McAndrews MP, Rovet J. Effects of early thyroid hormone deficiency on children's autobiographical memory performance. Journal of the International Neuropsychological Society: JINS 2013; 19 (4): 419-29.

15. Wheeler SM, Willoughby KA, McAndrews MP, Rovet JF. Hippocampal size and memory functioning in children and adolescents with congenital hypothyroidism. J Clin Endocrinol Metab 2011; 96 (9): E1427-34.

16. American Academy of P, Rose SR, Section on E, Committee on Genetics ATA, Brown RS, Public Health Committee LWPES, et al. Update of newborn screening and therapy for congenital hypothyroidism. Pediatrics 2006; 117 (6): 2290-303.

17. van der Sluijs Veer L, Kempers MJ, Maurice-Stam H, Last BF, Vulsma T, Grootenhuis MA. Health- related quality of life and self-worth in 10-year old children with congenital hypothyroidism diagnosed by neonatal screening. Child Adolesc Psychiatry Ment Health 2012; $6(1): 32$.

18. Azar-Kolakez A, Ecosse E, Dos Santos S, Leger J. All-cause and disease-specific mortality and morbidity in patients with congenital hypothyroidism treated since the neonatal period: a national population-based study. J Clin Endocrinol Metab 2013; 98 (2): 785-93.

19. Hassani Y, Larroque B, Dos Santos S, Ecosse E, Bouyer J, Leger J. Fecundity in young adults treated early for congenital hypothyroidism is related to the initial severity of the disease: a longitudinal population-based cohort study. J Clin Endocrinol Metab 2012; 97 (6): 1897-904.

20. Kreisner E, Schermann L, Camargo-Neto E, Gross JL. Predictors of intellectual outcome in a cohort of Brazilian children with congenital hypothyroidism. Clin Endocrinol (Oxf) 2004; 60 (2): 250-5.

21. Huo K, Zhang Z, Zhao D, Li H, Wang J, Wang X, et al. Risk factors for neurodevelopmental deficits in congenital hypothyroidism after early substitution treatment. Endocr J 2011; 58 (5): 355-61.

22. Bargagna S, Dinetti D, Pinchera A, Marcheschi M, Montanelli L, Presciuttini S, et al. School attainments in children with congenital hypothyroidism detected by neonatal screening and treated early in life. Eur J Endocrinol 1999; 140 (5): 407-13.

23. Connelly JF, Rickards AL, Coakley JC, Price GJ, Francis I, Mathur KS, et al. Newborn screening for congenital hypothyroidism, Victoria, Australia, 1977-1997. Part 2: Treatment, progress and outcome. J Pediatr Endocrinol Metab 2001; 14 (9): 1611-34.

24. Rovet J, Daneman D. Congenital hypothyroidism: a review of current diagnostic and treatment practices in relation to neuropsychologic outcome. Paediatr Drugs 2003; 5 (3): 141-9.

25. Kempers MJ, van der Sluijs Veer L, Nijhuis-van der Sanden RW, Lanting CI, Kooistra L, Wiedijk BM, et al. Neonatal screening for congenital hypothyroidism in the Netherlands: cognitive and motor outcome at 10 years of age. J Clin Endocrinol Metab 2007; 92 (3): 91924.

26. American Academy of Pediatrics AAP Section on Endocrinology and Committee on Genetics, and American Thyroid Association Committee on Public Health: Newborn screening for congenital hypothyroidism: recommended guidelines. Pediatrics 1993; 91 (6): 1203-9.

27. Salerno M, Militerni R, Bravaccio C, Micillo M, Capalbo D, Di MS, et al. Effect of different starting doses of levothyroxine on growth and intellectual outcome at four years of age in congenital hypothyroidism. Thyroid: official journal of the American Thyroid Association 2002; 12 (1): 45-52.

28. Simoneau-Roy J, Marti S, Deal C, Huot C, Robaey P, Van Vliet G. Cognition and behavior at school entry in children with congenital hypothyroidism treated early with high-dose levothyroxine. The Journal of pediatrics 2004; 144 (6): 747-52.

29. Jones JH, Gellen B, Paterson WF, Beaton S, Donaldson MD. Effect of high versus low initial doses of L-thyroxine for congenital hypothyroidism on thyroid function and somatic growth. Arch Dis Child 2008; 93 (11): 9404.

30. Mathai S, Cutfield WS, Gunn AJ, Webster D, Jefferies C, 
Robinson E, et al. A novel therapeutic paradigm to treat congenital hypothyroidism. Clin Endocrinol (Oxf) 2008; 69 (1): 142-7.

31. Albert BB, Heather N, Derraik JG, Cutfield WS, Wouldes T, Tregurtha S, et al. Neurodevelopmental and body composition outcomes in children with congenital hypothyroidism treated with high-dose initial replacement and close monitoring. J Clin Endocrinol Metab 2013; 98 (9): 3663-70.

32. Alvarez M, Iglesias Fernández C, Rodríguez Sánchez A, Dulin Lniguez E, Rodríguez Arnao MD. Episodes of overtreatment during the first six months in children with congenital hypothyroidism and their relationships with sustained attention and inhibitory control at school age. Horm Res Paediatr 2010; 74 (2): 114-20.

33. Rovet JF, Ehrlich RM, Sorbara DL. Effect of thyroid hormone level on temperament in infants with congenital hypothyroidism detected by screening of neonates. The Journal of pediatrics 1989; 114 (1): 63-8.

34. Rovet JF, Ehrlich RM. Long-term effects of L-thyroxine therapy for congenital hypothyroidism. The Journal of pediatrics 1995; 126 (3): 380-6.

35. Rodriguez S AV, Undurraga C EEDP Escala de evaluación del desarrollo psico motor de 0-24 meses. Editorial
Galdoc Santiago-Chile. 1989.

36. Haeussler IM MT. Test de desarrollo psicomotor de 2-5 años. Ediciones Universidad Católica de Chile Santiago-Chile. 1985.

37. De La Parra CA. Evaluaciones psicométricas del programa de seguimiento de niños con fenilquetonuria e hipotiroidismo congénito. Revista chilena de pediatría. 2008; 79: 103-9.

38. Lejarraga H MA, Menzano E, Fattore MJ. PRUNAPE: Pesquisa de trastornos de desarrollo en el primer nivel de atención. . Arch Argent Pediatr 2008; 106 (2): 119-25.

39. N O. Escala abreviada del desarrollo. Dirección General Colombia. 1999.

40. Pardo MGM, Edwards M. Test de desarrollo infantil TADI. Serie reflexiones: Infancia y Adolescencia Santiago: UNICEF. 2012.

41. Rivera González R, Sánchez C, Corral Guille I, Figueroa Olea M, Soler Limón K, Martínez Vázquez I, et al. Edad de presentación de los reactivos del Test de Denver II en Niños de 0 a 4 años de edad del Estado de Morelos. Salud Mental 2013; 36:459-70.

42. Bonnier C. Evaluation of early stimulation programs for enhancing brain development. Acta Paediatrica 2008; 97 (7): 853-8. 